\title{
ALgUNAS CONSIDERACIONES SOBRE "LO SENSIBLE" EN DE ANIMA
}

\author{
Rocio Mier y Terán \\ Universidad Panamericana
}

Here I argue that there can be found three different meanings of "sensible" in Aristotle's De Anima. These meanings suppose that it is better to understand sensation as activity more than as a passion.

La tradición aristotélica ha adoptado el término objeto para designar a lo sensible en relación al sentido; pues bien el objeto de los sentidos externos, lo sensible, aquello que especifica y constituye al sentido, es tratado por Aristóteles en distintos sentidos según su estatuto propio. Es decir, el objeto sensible, o más sencillamente, lo sensible no tiene en Aristóteles un solo sentido o significado, sino al menos tres distintos e irreducibles. ${ }^{1}$

Al inicio del libro II. cap. V se pregunta Aristóteles por qué los sentidos no dan lugar a la sensación en ausencia de objetos exteriores. La respuesta se limita a mencionar que es obvio que la facultad

1 Me refiero aqui al objeto de los sentidos externos y no al de los internos. Lo conocido por la sensibilidad interna no proviene sino de lo conocido por los sentidos externos.

Agradezco la colaboración del prof. José Luis Rivera por su ayuda en la elaboración de este artículo. 


\section{TOPICOS}

sensitiva no está en acto sino en potencia. ${ }^{2}$ El planteamiento al problema que tratamos de plantear no puede ser más sencillo: la sensación puede ser considerada en potencia o en acto. El sentido se encuentra en potencia respecto del sensible y se actualiza cuando se tiene la sensación. Entonces, tanto sentido como sensible se unifican en un sólo acto. Hay al menos tres sentidos de sensible: la cualidad física que actualiza al sentido, el acto pasivo ${ }^{3}$ de la afección sensitiva y la coactualidad de las formas en este acto.

El asunto reviste especial importancia. Lo que está en juego al tratar de "lo sensible" es el paso de lo sensible físico - la formalidad real accidental que existe en las cosas- a lo sensible en cuanto conocido - la forma intencional-. No entender esta conexión implica afirmar que la sensibilidad externa no tiene un estatuto propio, -porque o no se realiza en ella un acto cognoscitivo (como sugieren quienes trasladan el acto de la sensación hasta el sentido común), o porque este acto no llega a constituir una operación inmaterial (como sugieren otros que lo entienden como mera afección). ${ }^{4}$

2 De Anima 417a 14-20. En adelante, la numeración de Bekker que no indica el libro se refiere a esta obra.

3 Soy consciente de que esta expresión es contradictoria en el lenguaje aristotélico; pero más adelante se explica qué quiero decir con ella.

4 Buena parte de la literatura aristotélica parece inclinarse a minimizar el papel activo de la sensibilidad externa. Por ejemplo, Theodor EBERT, en "Aristotle on what is done in perceiving", Zeitschrift für philosophische Forschung 37 (1983) pp. 181-198 traslada la faceta activa de la percepción sensible hasta el sentido común. Por el contrario, algunos autores que reconocen : cierta continuidad entre las cualidades fisicas hasta la percepción sensible como T.W. BYNUM: “A new look at Aristotle's Theory of perception", History of Philosophy Quarterly 4 (1987), pp. 163-178 y respecto a Tomás de Aquino, Sheldon M. CoHEN: "St. Thomas Aquinas on the immaterial reception of sensible forms", The Philosophical Review 91 (1982) pp. 193-209 tienen algunas dificultades en entender que el último estadio de la sensación es un acto inmaterial por subrayar la pasividad del órgano. D.W. HAMLYN en sus notas a Aristotle's De Anima 
El "salto" que se pretende encontrar entre el conocimiento sensible y el intelectual no es mucho mayor al que se lleva a cabo desde la realidad fisica de la cualidad sensible hasta el sensible hecho uno con el sentido en la sensación. Tratare de mostrar que en De Anima hay suficientes elementos para creer que Aristóteles no incurre en los malentendidos mencionados y da una explicación coherente del tránsito de la cualidad fisica al conocimiento intencional.

El estatuto de lo conocido y el estatuto de lo real son distintos radicalmente para Aristóteles. Debe recordarse que en Metafísica excluye el ser veritativo del discurso porque es "una afección del pensamiento". ${ }^{5}$ En consecuencia, el problema que se plantea aquí es, en última instancia, de cómo hace Aristóteles para sostener que "lo sensible" es uno en acto con el sentido y al mismo tiempo mantener que el sentido externo está en potencia respecto de "lo sensible" externo.

Cuando Aristóteles trata del objeto del sentido establece tres modos distintos del "sensible", de esta manera logra explicar el "tránsito" de lo sensible fisico a lo sensible intencional.

1.- El primer sentido de lo sensible se refiere a la cualidad fisica. Cuando Aristóteles dice que "los sentidos no dan lugar a sensación alguna en ausencia de objetos exteriores", 6 y un poco más adelante,

Books II, III. Clarendon Press. Oxford 1968, pp. 99-105 no hace ninguna referencia al problema que planteamos. Las ideas aqui expuestas aparecen, en parte, tanto en Bynum como en Cohen, y también en Jaime ARAOS SAN MARTiN: "Pasión y conocimiento: lectura del De Anima de Aristóteles", Philosophica 16 (1994) pp. 119-123.

5 Met. VI 4, 1028a 35.

6 417a 3-4. Debo aclarar que el uso del término objeto es equívoco. Por un lado, en Aristóteles no aparece la expresión objeto sensible ni objeto exterior, sino el término aisthéton, literalmente "lo sensible"; y la 


\section{TOPICOS}

"porque los objetos sensibles forman parte de las realidades exteriores e individuales" 7 está reconociendo explícitamente que lo "sensible" no procede de la actividad misma del sentido, sino de las cosas externas que inmutan al órgano. "Lo visible, lo audible y el resto de los objetos sensibles son exteriores". ${ }^{8}$

Es necesario, por tanto, dar este primer paso que señala Aristóteles: el sentido puede ser considerado en potencia y en acto; y es llevado al acto por otra cosa en acto. ${ }^{9}$ La trillada sentencia de que nada hay en el entendimiento que no haya pasado antes por los sentidos se puede parafrascar para la sensibilidad externa de la siguiente manera: "nada hay en el sentido que no se refiera -al menos como origen- al sensible como cualidad fisica".

Está claro que para Aristóteles el sentido se encuentra en potencia respecto de su objeto considerado éste como cualidad. Bajo esta optica es válido afirmar que el sensible es sin el sentido, como cualidad fisica, por más que "lo oloroso sea precisamente el olor". ${ }^{10}$

2.- Afirma Aristóteles que la sensación parece ser un tipo de alteración. ${ }^{11}$ Es evidente que en el órgano del sentido hay inmutación; es decir, el órgano es "alterado" por el objeto externo considerado como cualidad sensible.

Es en este sentido en el que Aristóteles habla del sentido como potencia pasiva. Hay algunos textos que tomados aisladamente

expresión tà éxö, es decir. "las cosas de fuera". En adelante utilizo indistintamente "objeto sensible" $y$ "sensible" con esta salvedad.

$7417 \mathrm{~b} 27-28$.

$8417 \mathrm{~b} 20-21$.

9 Cfr. supra, nota 2.

10424 b 6 .

$11 \mathrm{Cfr} .415 \mathrm{~b} 25$ 


\section{EL “SENSIBLE" EN EL DE ANIMA}

pueden sugerir que se atribuye al objeto como cualidad en acto el principio activo del conocimiento, como cuando afirma que la sensación "tiene lugar cuando el sujeto es movido y padece una afección". 12

Hay que tener presente, para la interpretación del texto anterior, que es el mismo autor quién establece una analogia de proporción entre el alma (entelequia) y cuerpo con la facultad y el órgano: "si el ojo fuera un animal, su alma sería la vista". ${ }^{13}$ Esta es, desde luego, la entidad definitoria del ojo. El ojo, por su parte, es la materia de la vista, de manera que, quitada esta, "aquél no sería en absoluto un ojo a no ser de palabra". 14

El que el ojo no sea la vista significa que la inmutación que recibe según cierta proporción es la condición de posibilidad para que el sentido sea uno en acto con el sensible. $Y$ es que la forma fisica es recibida, asumida según el carácter propio del órgano, es decir, vitalmente. La forma es recibida no sólo pasivamente, sino al modo activo propio del viviente. Lo propio del viviente es asumir formas incorporándolas según su. propio ser. A eso se debe que toda la digresión del capitulo $\mathrm{V}$ se dedique a distinguir dos formas de pasividad: una puramente material y otra que implica formalidad, es decir, actualidad ${ }^{15}$

Según este aspecto el sentido es definido como "la facultad capaz de recibir las formas sensibles sin la materia al modo en que la cera

$12416 \mathrm{~b} 33-35$. Esta interpretación es ampliamente suscrita por COHEN.

13412 b 19-20. Hay una interesante discusión de este pasaje en Irving BLOCK: "Three German Commentators on the Individual Senses and the Common Sense in Aristotle's Psychology", Phronesis 9 (1964) pp. 58-63. Al margen de las conclusiones de Block, me parece que la interpretación de Baeumker, uno de los autores alemanes reseñados, es la correcta.

14 Cfr. 412b 22-23.

$15417 \mathrm{~b} 2-5$. 


\section{TOPICOS}

recibe la marca del anillo sin el hierro ni el oro: $y$ es que recibe la marca de oro o de bronce pero no en tanto que es de oro o de bronce". 16

Al afirmar que el sentido recibe formas sensibles sin materia se está refiriendo Aristóteles a lo que más tarde llamaron los escolásticos especie impresa. Es un modo de ser distinto de la forma fisica porque es recibido en el sentido según la indole propia del órgano.

Asi el objeto como cualidad sensible en acto inmuta al órgano del sentido pero adquiere en éste un nuevo estatuto: "es obvio que lo sensible [en el primer sentido] hace que la facultad sensitiva pase de la potencia al acto sin que ésta desde luego, padezca afección o alteración alguna" ${ }^{17}$

La alteración de la que trata el autor cuando se refiere a la que padece el sentido por el sensible se distingue de aquella que padecen los seres naturales "habrá que distinguir dos tipos de alteración. la una consistente en un cambio hacia estados pasivos y de privación, la otra hacia un estado activo, hacia su actividad natural". 18

La cualidad recibida en el órgano de acuerdo a la naturaleza de éste, es decir, la cualidad en el órgano es un modo peculiar de ser de lo sensible como afección, que no se puede confundir ni con la cualidad fisica, ni con el sensible en cuanto ya conocido.

Para Aristóteles el conocimiento es un acto de indole distinta al acto del movimiento transitivo. Es conocido el pasaje de Metafisica que ilustra esta diferencia:19 "Puesto que de las acciones que tienen limite

$16424 a 18-21$.

17431 a 4-5.

$18417 \mathrm{~b} 15-17$.

19 Met. IX. 6 1048b 23. Además, De Anima tiene un lugar paralelo: Cfr. 431 a 6-8. 


\section{EL "SENSIBLE" EN EL DE ANIMA}

ninguna es fin... acción (praxis) es aquella en la que se da el fin. Por ejemplo, uno ve y al mismo tiempo ha visto, piensa y ha pensado, entiende y ha entendido, pero no aprehende y ha aprehendido ni se cura y está curado". El conocimiento -también el de los sentidos externos- es un acto perfecto en cuanto que es simultáneo con el fin. El conocimiento es vida y por lo tanto actividad inmanente.

3.- El tercer "modo" de lo sensible que Aristóteles menciona no es otro sino aquél en el que el sensible es uno en acto con el sentido: "el acto de lo sensible y el del sentido son uno y el mismo". ${ }^{20}$ En el acto de conocimiento el sentido que se encontraba en potencia respecto del sensible es uno con él. Es aqui donde propiamente hay conocimiento -al ver tengo io visto- la operación y lo conocido se dan simultáneamente. La operación no produce el objeto, ni el objeto produce la operación, concurren en un sólo acto perfecto, el del conocimiento.

La forma fisica posee un estatuto distinto a la forma en cuanto conocida. Planteado el asunto desde lo sensible se puede afirmar que no es lo mismo lo sensible como cualidad fisica "exterior" a lo sensible hecho uno con el sentido en el acto cognoscitivo. ¿Qué es el color sino lo visto? Por eso Aristóteles dice que "lo oloroso es precisamente el olor", ${ }^{21}$ es decir, la forma cualitativa poseida en acto por la facultad. No es ni la pura cualidad externa ni la pura alteración, sino la forma de la cualidad con la forma del sentido, unidos en acto en la sensación.

Entre la forma cognoscitiva sensible y la forma cognoscitiva intelectual hay diferencias importantes (tantas, que sólo hay ciencia en el conocimiento intelectual); sin embargo, ambas formas son intencionales. No sucede lo mismo con la forma puramente sensible, ya que Aristóteles intenta explicar cómo se inicia el conocimiento en

$20425 b 26-27$.

21424 b 6 . 


\section{TOPICOS}

su nivel primario. La cualidad sensible y el sensible en cuanto conocido pertenecen a ordenes distintos.

La operación, el acto cognoscitivo no es propiamente causado por el objeto. No se puede reducir el conocimiento - aún el sensible- a la actividad fisica. La forma conocida es una en acto con el cognoscente. Es actividad inmanente, no transitiva; es praxis, no kinesis. Tampoco la operación produce el objeto. El sensible como conocido no es producto de la operación, ni ésta es producto de lo sensible fisico. $\mathrm{Si}$ así fuera, toda afección produciría sensación. ${ }^{22}$

Se podría tratar de inferir, a partir de estas consideraciones, que los tres modos de "lo sensible" son en última instancia tres "modos" del acto: el acto de la cualidad externa, la inmutación asumida vitalmente y el acto del conocimiento. Podria profundizarse en esta sugerencia, pero esto rebasa los limites del presente estudio.

22 En este sentido, la interpretación de COHEN a Tomás de Aquino parece un poco extraña. Cfr. op.cit., p. 195. Pero no es éste el lugar para discutir esta interpretación. 
Copyright of Tópicos. Revista de Filosofía is the property of Universidad Panamericana and its content may not be copied or emailed to multiple sites or posted to a listserv without the copyright holder's express written permission. However, users may print, download, or email articles for individual use. 